\title{
A proposal for a standard electronic anthropomorphic phantom for radiotherapy
}

\author{
J. S. Aldridge, P. J. Reckwerdt, and T. R. Mackie \\ Department of Medical Physics, University of Wisconsin-Madison, Madison, Wisconsin 53706
}

(Received 8 April 1999; accepted for publication 21 June 1999)

To create a database of human male and female computed tomography (CT) slices, the National Library of Medicine organized the "Visible Human Project." Since the male and female data sets provided are approximately $269 \mathrm{MB}$ and $915 \mathrm{MB}$, respectively, both the size and complexity have been reduced. While making the slices accessible to those with limited computing resources, the production of these reduced data sets also presents a unique opportunity to establish a standard human CT slice library for research in radiation therapy. A brief history of the original data sets is included, as well as details of the reduction process, applications to radiotherapy, and information on accessing these reduced image files. (C) 1999 American Association of Physicists in Medicine. [S0094-2405(99)02509-2]

Key words: Visible Human Project, computed tomography (CT), radiotherapy

The work presented is an extension of that begun by the National Library of Medicine's (NLM) Visible Human Project. One aim of this project was to create a universallyaccessible collection of CT data from a complete human adult male and female. ${ }^{1}$ However, the male and female image data provided by NLM are approximately 269 MB and $915 \mathrm{MB}$, respectively. The data sets were not acquired with a single field of view (FOV), and each set contains a few corrupted or missing slices. To produce a more manageable image database, the size and complexity of the data sets have been reduced. The benefit of such a data set lies in its applicability as a basis of comparison in dose computation and dose reconstruction, as well as in the development of optimization and registration (megavoltage and kilovoltage) algorithms for radiation therapy. While making the slices accessible to those with limited computing resources, the production of these reduced data sets presents a unique opportunity to establish a de facto standard human CT slice library for research and development purposes. A brief history of the original data set follows. Also included are details of the reduction process and information on how to obtain these image files.

The Visible Human Project has its roots in a 1989 NLM Board of Regents recommendation that "NLM should undertake a first project building a digital image library of volumetric data representing a complete, normal adult male and female." 1 Therefore, the Visible Human Project was created as a national resource for anatomical information. About 2 and 2.5 years later, respectively, appropriate male and female cadavers were procured. The male cadaver was that of a 38 year old, $199 \mathrm{lb}$., $5 \mathrm{ft} 11 \mathrm{in}$. man, while the female cadaver was that of a 59 year old, $5 \mathrm{ft} 5.5 \mathrm{in}$. woman. ${ }^{2}$ Shortly after death, each cadaver was perfused with approximately 191 of $1 \%$ formalin (a type of preservative/fixative used for tissue specimens) and an anticoagulant to retard deterioration. The cadavers were placed horizontally on their backs in a plywood box and surrounded with a foaming agent to maintain a fixed position. For fiduciary markers, two $3 \mathrm{~mm}$ O.D. Tygon tubes filled with $4 \mathrm{mmol}$ copper sulfate solution and 5\% Omnipaque (I) were attached with Liquid Nails to the skin surface from head to toe. ${ }^{3}$

The original male data set consists of 509 axial CT scans

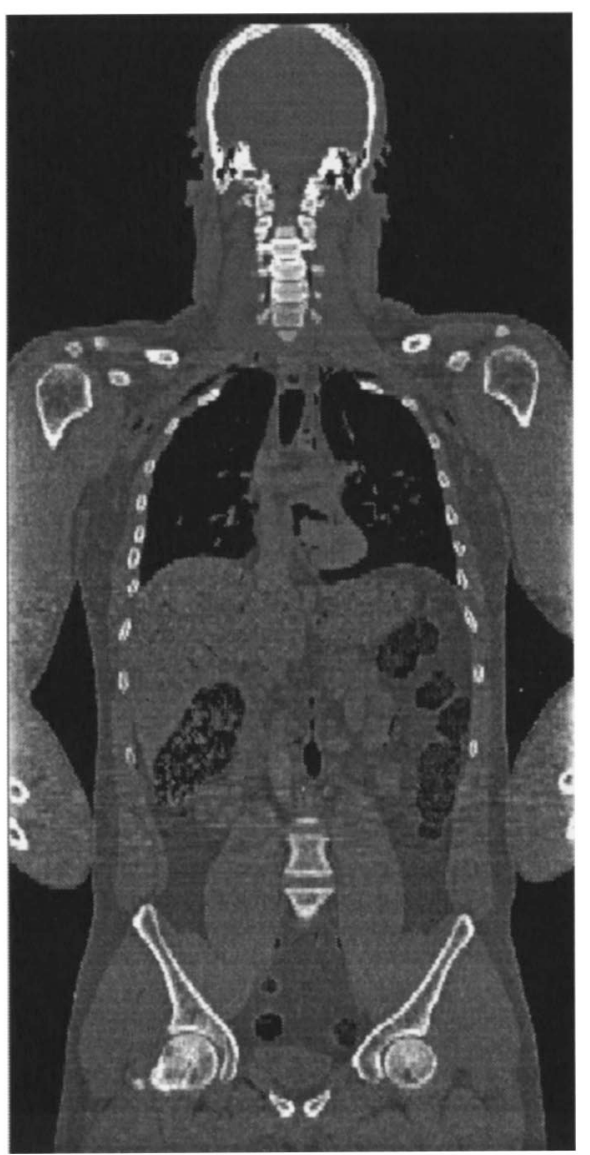

FIG. 1. Coronal view of central slice from the Visible Human Male reduced data set. 


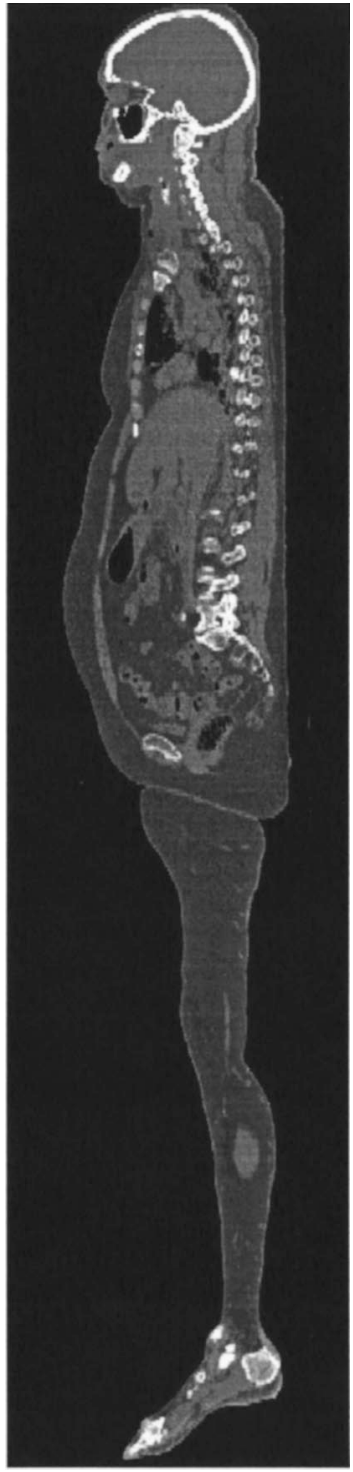

FIG. 2. Sagittal slice from the Visible Human Female reduced data set.

of the body taken at $1 \mathrm{~mm}$ intervals for most of the head slices, $3 \mathrm{~mm}$ intervals from approximately the mandibular to the pelvic regions, and $5 \mathrm{~mm}$ intervals throughout the lower extremities with a resolution of $512 \times 512$ pixels. The data set from the female cadaver has the same characteristics as the male cadaver except that the entire body scan was collected in $1 \mathrm{~mm}$ increments, resulting in 1733 images. In the male data set, approximately 84 scans corresponding to a segment of the leg ranging from a little above the knee to the ankle were never acquired. While in the process of obtaining these scans, there was a serious concern that the cadaver might be in danger of decomposition. So, this particular segment was ignored in order to get the body into the freezer and not jeopardize the ability to obtain cryosections. ${ }^{3}$ For the female cadaver, no deterioration problem was encountered. However, the first half of the scans was acquired with the cadaver entering the scanner head-first, whereas the body was scanned feet-first for the last half. As a result, a slight align-

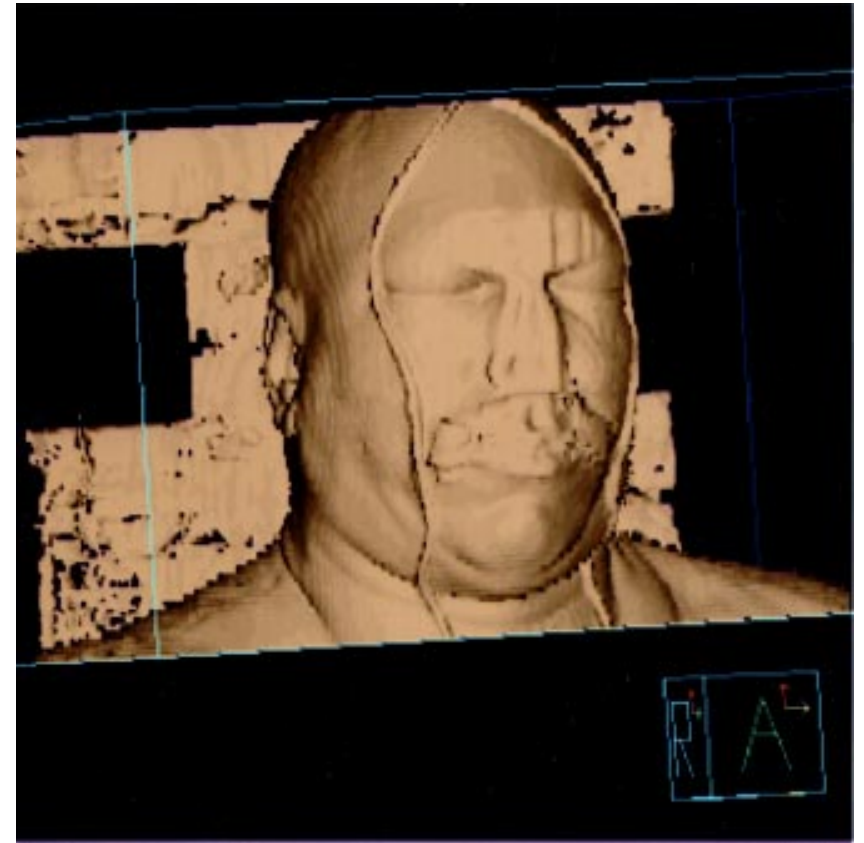

FIG. 3. Three-dimensional volume rendered representation of the treatment field.

ment discrepancy was introduced creating an obvious discontinuity between the halves.

Since the male and female original data sets are approximately $269 \mathrm{MB}$ and $915 \mathrm{MB}$, respectively, they were reduced in the following manner. First, the headers were removed, and all slices were scaled to a $460 \mathrm{~mm}^{2} \times 460 \mathrm{~mm}$ FOV. In the original CT slice data, the scans were not all

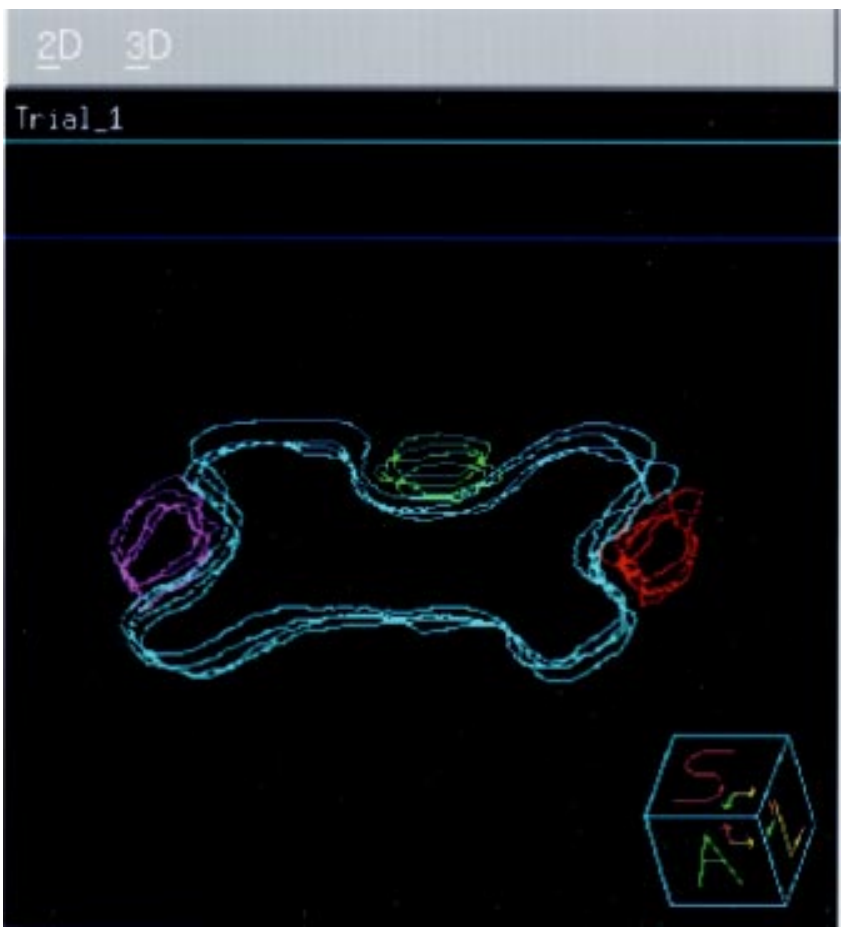

FIG. 4. Contours of left parotid (red), right parotid (magenta), spinal cord (green), and tumor region (blue). 
obtained with the same FOV but varied from $250 \mathrm{~mm} \times 250$ $\mathrm{mm}$ to $480 \mathrm{~mm} \times 480 \mathrm{~mm}$. A $460 \mathrm{~mm} \times 460 \mathrm{~mm}$ FOV was chosen simply because the bulk of the "Male" scans was collected with such an FOV. For the sake of consistency, the same FOV was applied to the "Female" data set. Next, a bilinear interpolation algorithm was applied to reduce the image sets to $256 \times 256$ pixels, thereby decreasing resolution by a factor of 2 . Due to voxelization effects, this change in resolution results in slight modifications of pixel values in high gradient regions only. Otherwise, no significant differences in information provided by the reduced data sets and the original scans have been noted.

Since only a few ( 3 or 4 ) of the $1 \mathrm{~mm}$ and $3 \mathrm{~mm}$ slices were corrupted, each corrupted slice was replaced with the slice that lay just above it. Finally, simple slice averaging reduced the storage for the male and female data sets to 41 $\mathrm{MB}$ and $76 \mathrm{MB}$, respectively. For both image sets the $z$-resolution (slice width) is $3 \mathrm{~mm}$ throughout all of the body segments. Due to the change in positioning of the female cadaver halfway through the scan, the slices that were acquired "feet first" have been adjusted by approximately 5.4 $\mathrm{mm}$ in both $x$ and $y$ in the reduced data set to obtain correct alignment. Also, as a consequence of the problem noted above that prevented a significant portion of the Visible Human Male leg slices from being collected, only the upper torso reduced set (41 MB) has been provided. Since all of the Visible Human Female CT slices were obtained, the reduced data set for the entire body is provided (76 MB).

The reduced slices are available for downloading at the following web site: http://www-madrad.radiology.wisc.edu/ vishum/index.html. These are fundamental data sets that will be quite useful for the radiation therapy community. They may form a basis for standardization of radiotherapy dose calculations and optimization comparison studies. Unfortunately, the conversion curve that relates $\mathrm{CT}$ number to electron density is not available for the Zeus:CT HiSpeed Adv scanner that was used to gather the Visible Human data. However, on the web page mentioned above, it is arbitrarily proposed that the curve generated for the University of Wisconsin-Madison's radiotherapy Siemens Hi-Q CT scanner be used as a substitute. Comparisons of measured physical densities from the Visible Human scans with those values obtained from "live" patient data for lung and several different soft body tissues show no significant dissimilarities that would preclude the applicability of the Visible Human CT data to living tissue CT data.

Included in Figs. 1 and 2 are representative coronal and sagittal views from the Male and Female reduced data sets, respectively. Figures 3-5 show the development of an intensity-modulated radiotherapy plan for treatment of nasopharyngeal carcinoma using slices from the Male reduced data set. Figure 3 is the $3 \mathrm{D}$ volume rendered representation

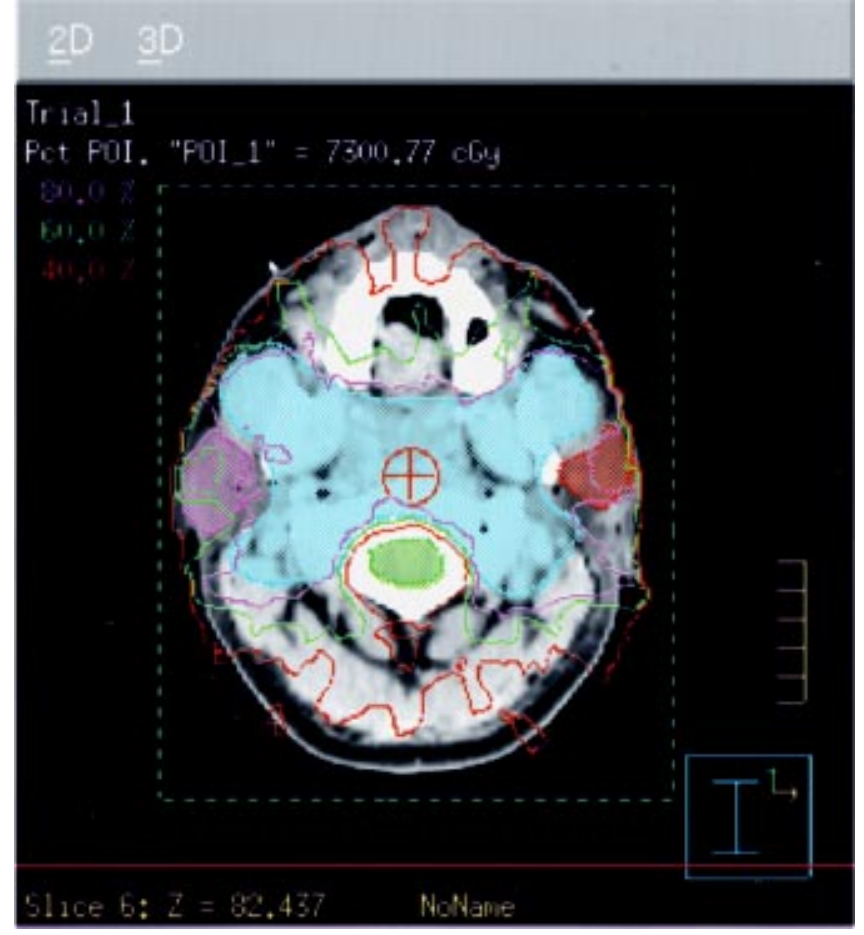

FIG. 5. Isodose lines from an intensity-modulated radiotherapy plan for treatment of nasopharyngeal carcinoma.

of the treatment field. After obtaining the CT slices, the critical structures and tumor volume(s) are contoured. As shown in Fig. 4, the left parotid (red), right parotid (magenta), spinal cord (green), and tumor region (blue) are the primary regions of interest (ROIs). The final figure (Fig. 5) shows the ROIs overlaid on the CT image along with isodose lines from the experimental intensity-modulated delivery.

A single License Agreement has been obtained from the Visible Human Project/National Library of Medicine allowing access to CT scan data from both a male and a female cadaver. This scan data has been reduced in both size and complexity producing a more manageable image database. Work is in progress to produce equivalent MRI image sets from the data provided by the Visible Human Project thereby also allowing for image fusion research. Although the production of these reduced data sets does not constitute a scientific discovery or engineering development, it may provide the basis for standard electronic CT slice libraries of a human male and female not only for strictly educational purposes but also for radiation therapy research and development.

\footnotetext{
${ }^{1}$ Visible Human Project Web Site: http://www.nlm.nih.gov/research/ visible.

${ }^{2}$ Electronic mail: vhp@nes.nlm.nih.gov

${ }^{3}$ V. Spitzer, M. J. Ackerman, A. L. Scherzinger, and D. Whitlock, "The Visible Human Male: A Technical Report,' J. Am. Med. Assoc. 3, 118 130 (1996).
} 